\title{
Rehabilitation Programme to Promote Task Relearning and Generalisation after Stroke: A Review of Literature
}

\author{
Karen PY Liu ${ }^{1 *}$, Chetwyn CH Chan ${ }^{2}$, Nikki Tulliani ${ }^{1}$, Marcus $\mathrm{CK} \mathrm{Ng}^{1}$, Leonard SW Li ${ }^{3}$ \\ ${ }^{1}$ University of Western Sydney, Australia \\ ${ }^{2}$ Applied Cognitive Neuroscience Laboratory, Department of Rehabilitation Sciences, The Hong Kong Polytechnic University, Hong Kong \\ ${ }^{3}$ Tung Wah Hospital, Hong Kong
}

*Corresponding author: Karen Liu, Associate Professor, University of Western Sydney, Locked Bag 1797, Penrith NSW 2751, Tel: +61-2-4620-3432; Fax: +61-2-4620-3792; E-mail: Karen.Liu@uws.edu.au

Received date: June 29, 2014, Accepted date: Aug 12, 2014, Published date: Aug 18, 2014

Copyright: (C) 2014 Liu KPY, et al. This is an open-access article distributed under the terms of the Creative Commons Attribution License, which permits unrestricted use, distribution, and reproduction in any medium, provided the original author and source are credited.

\begin{abstract}
Background and Purpose: The relearning of functional skills following a stroke is an essential part of the rehabilitation process. Rehabilitation post stroke may facilitate an individual to return to independent living. However, the skills learnt during this process do not necessarily transfer to the skills required for daily functioning. This review addresses the issue of generalisation of skills learnt by discussing the connectionist model.

Summary of Review: Connectionism models how the human brain functions and stipulates that the units in an input layer feed their activations forward to the units in the hidden layer for interpretation and then to the output layer for execution. These units are connected and distributed in a connectionist network. The activation of clusters of units in retrained tasks will provide signals to other different but connected tasks that have not been retrained. Adopting the concept of the connectionist model, the relearning of tasks after brain damage enhances the relearnt skills by transferring them to other tasks that share similar units within the same connectionist network, resulting in generalisation. Research evidence has shown that the strategies of self-regulation and mental imagery further enhance the relearning and generalisation of skills among people with brain damage. By identifying a list of daily tasks that fall within a connectionist network and the appropriate use of training strategies, the skills developed during the rehabilitation process could lead to effective task generalisation to suit the needs of independent living and community re-integration of the individual.
\end{abstract}

Conclusions: The Connectionist Model provides a theoretical base for developing evidence-based interventions throughout the acute, rehabilitation and community phases. The Connectionist Model is the theory by which the cognitive skills learned to perform one particular behaviour, or skill, are transferable to executing other similar skills or beahviours without specifically relearning those skills or behaviours.

Keywords: Rehabilitation; Occupational therapy; Relearning; Generalisation; Stroke

\section{Introduction}

Following a stroke an individual may experience cognitive, physical and psychological deficits. Their deficits extend from acute suffering to long-term disabilities in re-integration into the community. Existing interventions aim to stabilize medical conditions in the acute phase, and to retrain the previously learnt tasks through actual trial and performance in the rehabilitation phase. The common outcome indicators are mortality rates, length of hospital stay, rehabilitation cost, hospital discharge location and functional status on discharge [1-5]. The important factors, such as long-term adaptation and community re-integration, however, are equally important as they reflect the quality of life of the patient [6]. There is a lack of evidence supporting the effectiveness of relearning previous learnt tasks and hence generalisation to other tasks not practiced during the rehabilitation process. The theoretical base of the Connectionist Model is important for supporting evidence-based practice, however remains unclear.
This paper reviews the problems encountered in the training of previously learnt skills in people with brain damage. It discusses the usefulness of connectionist theory in modeling the relearning and generalisation of previously learnt skills in individuals with brain damage. Illustrations depicting the principles and mechanisms by which the connectionist theory can be employed into standardised interventions for individuals with brain damage in the acute, rehabilitation and discharge management phases.

\section{Functional Regain of People with Brain Damage}

Functional regain depends on the extent to which the damaged brain cells regenerate and functionally reorganise after active learning. Studies on animals such as rats with visual deficits demonstrate the plasticity and regeneration characteristics in the damaged brain structure and transynaptically in the connected structures $[7,8]$. Another study found that cutting part of the connections between the visual association and the frontal cortex did not affect a visual discrimination task that was well learnt by instrumental conditioning prior to cutting the connections [9]. This supports the hypothesis that other connections were established as a substitution for the originally damaged pathways and to communicate the input stimuli to produce 
the output behaviour. This reconnection involves neuronal reorganisation and repair of the damaged neural network. Motor skill training of rats with unilateral damage to the sensorimotor cortex resulted in an increase in synapse to neuron ratios in the intact motor cortex when compared with the control group [10]. This is further demonstrated by another study that shows the regaining of hand functions in 15 patients post stroke was accompanied by reorganisation of the primary somatosensory cortex that provoked a transient enlargement of the hand representation [11]. These findings illustrate the regeneration activities of the brain after brain lesions in both animals and humans.

Other studies have put forth the notion that active learning plays an important role in enhancing the plasticity of the brain $[12,13]$. In one study, people with global brain damage resulting from neonatal alcohol exposure were engaged in a therapeutic motor training intervention. The findings indicated that active involvement in learning appears more effective than interventions that merely allow the subjects to passively engage in activity [13]. This is also supported by a recent study using active cueing and the auditory-motor processes than the more passive stimulation to enhance neuro-plasticity and motor learning in people post stroke [14].

Therefore, regeneration of brain cells after brain damage is essential for the regaining of function, and the active participation in the learning process of the regaining of function can facilitate such cell regeneration. In other words, synaptic regeneration of the damaged brain structure and functional reorganisation of new or recovered brain cells are essential for people with brain damage to recover or expand behavioural depth.

\section{The Connectionist Model}

The Connectionist Model, first developed by Warren McCulloch and Walter Pitts in 1943, is based on a neural metaphor to describe the structural mechanism of how the brain works. The neural network model is applied in the Connectionist Model to simulate the human brain by building units that are joined by connections and arranged into layers $[15,16]$. The Connectionist Model is described here to explain the functional reorganisation and the potential for transferring generalised skills relearned and regained through rehabilitation to other skills and behaviours that have not specifically been retrained or relearned.

Under the connectionist model, several terminologies are used to describe the pathways of the neural network model by which the brain is assumed to function. These are; (a) Units known as neurons; (b) Activation of a unit, at each point in time, brought about by action potential; (c) Connections between units, known as synapses (i.e. synapses of neurons), which contain connection weights, that determine the extent to which the action of one unit affects the action of another unit or units; and (d) Learning rules, which change the network's behaviour by altering the weights of its connections (transmission of action potential and the strength of synapses). In the connectionist network, units are organised into separate layers: the input, output and hidden layers. The central assumption is that, after receiving the stimulations, the units in the input layer feed their activations forward to the units in the hidden layer for interpretation. The signals are then fed to the output layer for execution. In other words, when the dendrites of a neuron are activated by a stimulus, it sends a message down the axon to the terminal buttons where the neurotransmitter is released, causing excitation of the interneuron.
The stimulation of the external environment, which can excite or inhibit, activates the units in the input layer. The units receiving excitatory signals are activated, whilst those receiving inhibitory signals remain inactive. These active and inactive units form the pattern of activations in the input layer. The activations are then propagated to the units in the hidden layer for processing.

The connection weights of the units also play an important role in learning. The extent of activation between units in the connectionist network is determined by the connection weights, creating different behaviours. A connectionist network contains sets of units with different patterns of activation, that gives rise to different sets of similar behaviours (Figure 1). Different input units are selected and activated to create different output behaviours. The relevant units in a network that constitute certain behaviour are thus 'distributed' in the network $[15,17]$. For example, a person with brain damage has relearnt to use a telephone (behaviour A), or how to operate an automatic teller machine (behaviour B). The performance of behaviour A occurs by the activation of the input units 1 (communication), 5 (pressing numbers) and 7 (problem solving): because of this distributed representation of the units in the connectionist network, behaviour B can be easily elicited due to the ready activation of units 5 and 7 . The learning of one behaviour promotes the learning of another behaviour, through the shared units that are common in the Connectionist Model. Generalisation of functions thus occurs.

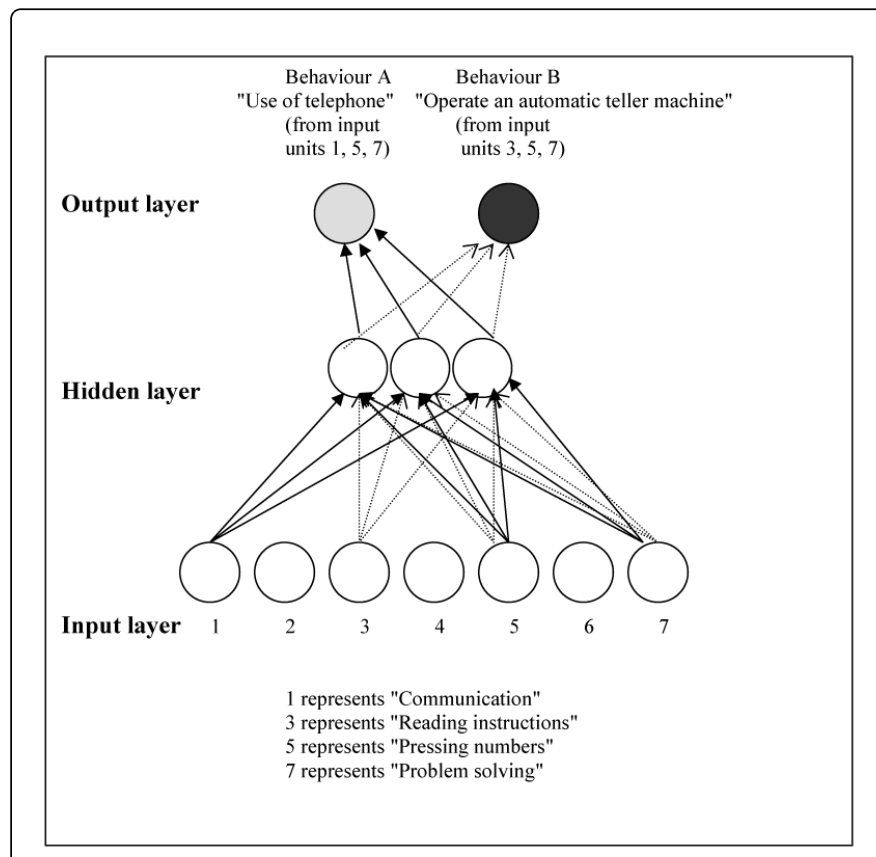

Figure 1: Schematic representation of a connectionist network with two target behaviours

\section{Learning Under the Connectionist Model}

Most of the studies on the application of the Connectionist Model are based on the recovery of language functions following brain damage $[18,19]$. The network was first trained so that the equations explaining the learning pattern were set. The trained network was then purposely damaged by removing a specified proportion of the 
connections between two groups of units, leading to a loss of the ability to recognize words previously learnt by the system. The network then underwent training on relearning the words. The results demonstrated that learning occurs within the same connectionist network for both trained and untrained words. It demonstrated generalisation of functions within the same connectionist network.

Unlike novice behavior, learning of previously learnt behaviors undergoes a slightly different process under the connectionist model. The previously learnt experience facilitates the relearning of the behavior due to the existence of a set of connection weights stored in the network. The facilitation is achieved by giving the individual the background knowledge for future learning. This accelerates the relearning of the output behavior, thereby reducing the time taken to achieve a particular competence level. This phenomenon is very important for individuals with brain damage who undergo rehabilitation.

\section{Network Breakdown after Brain Damage}

Previous experiences may be affected in a damaged brain, due to the breakdown of the connections in a network. This results in the loss of previously learnt behaviors and skills. This connection breakdown may be due to two reasons. The first happens when a behavior is learnt but not practiced for a while: the connection weights within the network may be degraded so that the regularities of the input and output patterns are no longer maintained. This is referred to as 'decay'. Another reason is the damage of the network that occurs in brain damage, which causes a breakdown of connections or damage of a subset of the units in the network due to the disrupted value of the connection weights. This leads to the loss of information or breakage of information transmission. The remaining information is incomplete and disconnected when trying to elicit the desired behavior. Both conditions may result in an inability to elicit the same output behavior with the same input stimuli, or in the worst case, a complete loss of the previous learnt behavior or skill.

\section{Relearning after Network Breakdown}

Relearning a behavior refers to the reviving of a previously learnt behavior that has been disrupted by brain damage. It involves reestablishing the connection weights or synapses within the network, diverting the information by building new connection weights or synapses, or activating the units or neurons that have not previously been used.

According to the model, the connection weights between units, and patterns of activation within the units in a layer, are stored in a "filing cabinet". The access and retrieval of a particular set of connection weights and patterns of activations on the request to perform a behavior occur in an intact system. The easy searching of information in the "filing cabinet" helps to speed up the process of propagation of activations among units in such a way that the connection weights are at a level that decreases execution time. This is termed content addressable memory [20]. After brain damage, the system is disrupted with part of the memory in the "filing cabinet" being erased. Only fragments of information remain in the system. The extent of the memory loss, or loss of connection weights, and thus the accessibility of information, depends on the severity of the damage. During the rehabilitation the connection weights of these units are reestablished, or in other words, the "filing cabinet" is rebuilt. This will then allow the relevant units to be linked together to elicit the previously learnt behavior.

Information processing when relearning a previously learnt behaviour in a damaged connectionist network is similar to the steps in Bloom's taxonomy of learning (Figure 2) [21,22]. They are comprehension, application to existing situation, analytical reasoning to tease out the relevant information, synthetic reasoning to collate the related parts with the past experience, and evaluation with reference to the present situation. The final decision constitutes the patterns of activations of the units in the output layer, which is the final behavior registered. At this point, when the patterns of activation of the units in all the layers are established, the strengths of the connections among the units are sufficiently set when the behavior is learnt and remembered. Those units that are strongly connected have larger connection weights and are adjusted through the activations propagated among units until a desired output behavior is achieved.

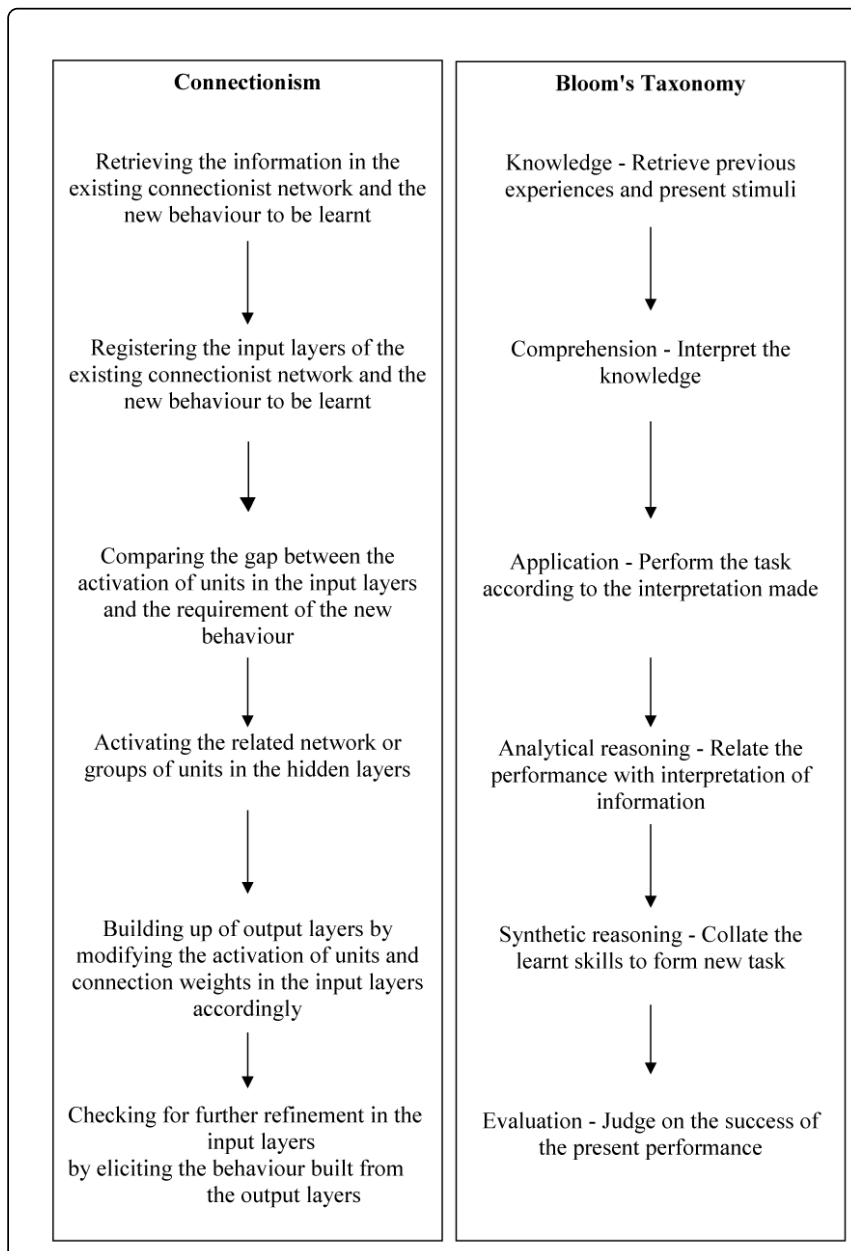

Figure 2: The learning model under connectionism and Bloom's taxonomy

Learning by repeated practice without external feedback takes time, and the output behavior may deviate from the external standard. The success of this unsupervised learning also depends on the ability of the individual to recognize their own shortcomings and adjust their behavior accordingly $[23,24]$. On the contrary, when the skills are learnt with supervision, that is, under guided training, the person 
learns with external feedback. The feedback is a form of reinforcement, as in instrumental conditioning [25], providing stimulation as a form of input signal that helps to speed up the regulation of the patterns of activations and thus the weights of the units. If training and feedback are provided in all six stages of learning, as suggested by Bloom's taxonomy, the activations and weights may change towards the desired output behavior at every stage of learning in response to the feedback given. Hence, the output behavior can be achieved within a shorter time frame and is comparable with the external standard under supervised learning $[23,24]$.

\section{Generalisation}

Generalisation is the ability of a person to maintain the skills learnt over time. It demands that a person perform a skill learnt in one situation and apply it in a variety of other situations or contexts [26]. In the context of rehabilitation of individuals with brain damage, the skills relearnt after brain damage are required to be generalized to other functions and situations after discharge from the health care institution $[27,28]$. The extent of Generalisation will determine the success of the person's re-integration into the community.

Generalisation can briefly be divided into three types: response maintenance, stimulus Generalisation, and response Generalisation [26]. Response maintenance refers to the tendency of a learnt behavior to be repeated over time. Stimulus Generalisation requires a response trained in a particular setting to be modified and assimilated in a different setting. For example, the use of automatic teller machines and vending machines are two different behaviors with the similar nature of operating a machine. During the performance of the new target behavior, the network may undergo a process of retrieving past experience and skills acquired from the already-learnt behavior. This retrieval of information guides the selection of appropriate units and patterns of activation among the units for the new target behavior, and finally reestablishment and synchronization of connection weights and task performance patterns to elicit the new behavior. The success of Generalisation enhances an individual's functioning, particularly for those with brain injury.

\section{Clinical Strategies for Connectionist-based Generalisation}

In a damaged network, the rebuilding of discrete sets of damaged units should take place before the rebuilding of the whole network. This phenomenon is in line with the hierarchical order of complexity in learning, as simple learning is accomplished before more complex learning. For example, learning one-digit addition helps to build up the ability to do two-digit addition work. To facilitate the establishment of these building blocks, the individuals have to identify the steps of these blocks. This would require the individual to analyse and divide the whole behavior into different logical steps and link them together to compose the behavior. This is known as chunking, which is a preparatory strategy to facilitate the relearning of discrete steps at a later stage $[27,29,30]$. The person has to understand the whole task performance by either reviewing the previous experience or by observing others doing it before the individual steps can be composed. Perceived problems in the task performance can then be identified by the individual. The process of chunking can be improved by feedback and guidance in which the logical steps identified are reinforced and the process of identifying the problematic steps and seeking solutions for each of the problematic steps by the individual is facilitated.

Self-regulation is the second strategy to facilitate behavior generalisation [31-33]. This strategy is particularly useful for the rebuilding of discrete damaged groups of units identified by chunking, because it requires the individual to relearn the patterns of behavior in the individual steps of a behavior. This involves a sequential practice of the steps to relearn the problematic skills leading to the reestablishment of proper connections or reactivation of the inactive units in the network. After self-regulation, the proper connection weights of the discrete steps of a damaged network are reestablished. This process helps to rebuild the damaged connectionist network, hence forming the basic foundation for the Generalisation of behavior. The foundation of solving the existing problems encountered by the individual, and practicing solving those problems, will be stored as connection weights and patterns of activations within networks that are available for retrieval in the future.

Generalisation of behavior does not occur under the self-regulatory strategy. Instead, it is the third strategy, mental imagery, which is a form of vicarious learning in which the individual forms a mental representation of the task performance under strategic control [34, 35]. Mental imagery is a process in which an individual processes the behavior mentally as if taking part in the actual performance of that particular behaviour. By doing so, the process of synchronisation of the relevant groups of units is initiated and thus the connection weights and patterns of activations required for the behaviour are further strengthened. The simulation and mental process would explicitly elicit the constraints, both physical and mental, of the human body associated with the task performance [27, 28, 30, 36, 37]. These constraints are the realisation of the products of the previous chunking and self-regulation strategies undertaken by the individual. They facilitate the linking together among the relevant groups of units that are necessary to form the behavior within the same connectionist network (response Generalisation). Successful mental imagery depends greatly on encoding the requirements of the target behavior, activating the somatomotor representation of the body, and actively comparing the actual behavior demands and the available abilities and bodily constraints $[27,28,30,38]$.

Numerous studies have investigated the actual brain activities involved in mental imagery, using imaging techniques [39-41]. These findings support the notion that the cortical areas activated during the mental imagery of a movement coincide with those activated during the actual performance of a task. This phenomenon is demonstrated by one of the studies, which compared the localization of neural activity in human brains during mental imagery with the real motion of the task [40,42-44]. The results suggest that mental imagery is accomplished within the same specialised areas of the brain that are held responsible for the real motion of a task.

Through mental imagery, the same process in the connectionist network occurs as in the actual practice of task performance. The connectionist network is regulated and the relevant synapses are strengthened. Mental imagery enhances Generalisation of the skills learnt. With the help of chunking and self-regulation to facilitate the learning of the lost discrete skills after damage to the brain has occurred, the effectiveness of mental imagery is enhanced. Mental imagery reinforces the established connection weights in the network during response maintenance. Task performance can therefore be maintained. During stimulus Generalisation, mental imagery helps to transfer the established connection weights of a behavior to another 
setting. The strengthening of the connection weights during mental imagery help to transfer the behavior even with possible distracting stimulations in another setting. Mental imagery also facilitates the reestablishment and synchronization of relevant connection weights necessary for another behavior with a similar nature to the already learnt behaviour. The phenomenon of activating the similar brain structures as in actual practice explains the use of mental imagery in transferring the learnt skills to another behavior.

\section{An Intervention Programme for People with Brain Damage}

The intervention programme has been built on the theory that a connectionist network connects the units that are common and responsible for the performance of a group of tasks. The training on selected connected tasks after damage to the brain helps to rebuild the units and hence the entire network. In the clinical trials, it was demonstrated that the programmer could promote relearning and Generalisation of the lost function post stroke [27,28,30]. They supported the hypothesis that connection weights and patterns of activations of the units associated with the relearnt tasks propagate to those of the non-relearnt tasks.

\section{Task Generalisation Training}

The training used in this programme is a sequential, carefully selected cluster of tasks which theoretically fall within the connectionist network. The tasks are categorised according to their task component and complexity. Task components are analysed with respect to three parameters: sensori-motor, verbal-spatial-cognitive and emotional-coping. The sensori-motor factor represents the ability to integrate sensory information such as the visual and tactile, which provides the feedback necessary for precise and, purposeful voluntary motor movement. The verbal-spatial cognitive factor refers to the ability to receive, think, process information and to express oneself. The emotional-coping factor is the adaptive behaviour and adjustment due to one's own body constraints. Those tasks that share similar components are regarded as falling within the same connectionist network, and are selected for the training protocol.

The tasks used in the treatment programme are the daily tasks which are particularly needed for the independent functioning of individuals with brain damage. Tasks such as eating, transferring from one chair to another, taking a bath and going to the toilet are regarded as sharing similar components, such as; hand grip, visual perception, eye-hand coordination, weight shifting and balance, and hence are within the same connectionist network (Figure 3). For instance, "eating a meal" and "transferring from one chair to another" are composed of fewer steps and demand fewer functional components than "taking a bath". Hence, the former two tasks become the "foundation tasks" and the building blocks of the latter, which is termed the "prospective task".

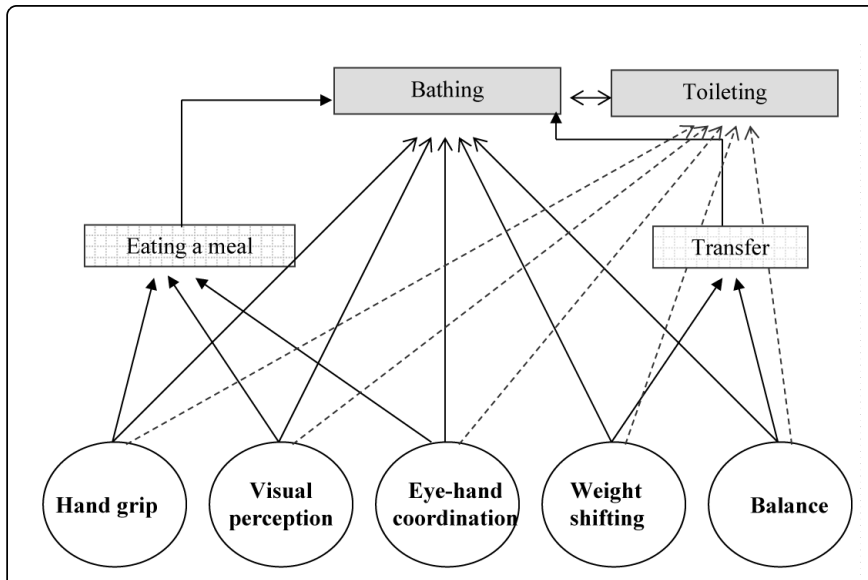

Figure 3: Schematic representation of three tasks using the concept of the connectionist model

By using the chunking and self-regulation strategies, the steps of performing "eating a meal" and "transferring from one chair to another" are reviewed. The problems encountered in their performance due to brain damage can be identified. The task performance is therefore relearnt and hence the connectionist network is reestablished. Since the tasks fall within the same connectionist network, by training the individuals to relearn the foundation tasks, this rebuilding of the network can enable individuals to maintain the task performance of "taking a bath" as well as to generalise the skills re-learnt to other similar but different tasks like "going to the toilet" in this example.

\section{Different phases of the training programme}

There are two stages in task generalisation training (TGT). The first stage makes use of the strategies of chunking and self-regulation for relearning the skills of the "foundation tasks". The second stage uses the relearnt tasks together with the mental imagery to develop the competent performance of the prospective tasks. The tasks used for stage one basically demands less of individuals in terms of their verbalspatial-cognitive, sensori-motor and emotional-coping abilities. Those in the final stage of TGT should be composed of the components necessary for the most difficult tasks that the individuals may face in their daily lives. For example, eating a meal demands less in all three aspects when compared with taking a bath. Eating a meal is therefore a task used in stage one, whilst taking a bath is used in stage two.

By the end of stage one, the individuals manage to relearn the "foundation tasks" by using chunking and self-regulation strategies. In stage two, mental imagery begins on "prospective tasks", which are more difficult and complicated to perform. In the above example, the "prospective tasks" such as "taking a bath" involve a higher level of physical and cognitive abilities and more steps than the "foundation tasks" such as "eating a meal" and "transferring from one chair to another". Other examples of "prospective tasks" are going to the toilet, and using telephones and automatic teller machines. It is also common that the "prospective tasks" are more geared toward independent living in the community. To facilitate learning and mental imagery, the pre-requisites are the ability to maintain attention, comprehend information, and sit upright. 
TGT can commence during the acute phase, in which individuals are monitored on their cognitive abilities such as attention and comprehension. For example, an individual who is able to communicate with others but is not ready to sit upright due to their unstable medical condition will practice chunking by breaking down the task of "eating a meal" into sequences. Video display of the task performed by others will be used to help the individual to retrieve their memory of the task performance. They can then be guided to identify problems encounted in these tasks after suffering damage to the brain, as a precursor to the self-regulatory strategies. During this step, display cards with audio input may help the individual to identify probable problems in each step. This may facilitate the individual to realising their difficulties engaging in tasks, and prompt active participation in their rehabilitation.

Once the individual has achieved a stable medical condition, they can then proceed to using the self-regulatory strategy. They will be asked to perform the tasks whilst video-taping. By looking at their own performance on video and using the self-regulatory strategy, they are prompted to work out the particular steps that need to be taken to address the problems, and subsequently discover ways to successful performance. With these in mind, the individual then performs the task of "eating a meal" again with the self-corrected methods. Again, depending on the individual's condition, the tasks that they can cope with, demanding fewer physical and cognitive abilities, are practiced first. In this case, with adequate sitting balance and the use of both upper extremities, the individual will start with simple "transferring" tasks. The tasks that the individual practices will increase in difficulty as their functional ability improves. These form the foundation tasks that will provide the individual with the necessary verbal-spatialcognitive, sensory-motor and emotional-coping abilities to proceed to the prospective tasks of "taking a bath" and "going to the toilet", which are critical to independent functioning for discharge to the community.

When the individual is able to handle self-regulation, they will be introduced to mental imagery. During mental imagery, the individual is also required to imagine them self performing the tasks with the effects of their body constraints by reporting the temporal components of the tasks. The methods of coping with these constraints using the self-regulatory strategy learnt earlier are put into the mental imagination of the task performance. Picture cards that show the beginning and last steps of the tasks may help the individual in the mental imagery process. After the missing steps and solutions are identified by the individual, the task is mentally imagined by the individual again. During this process, the individual will go through the whole task by performing it mentally. Actual task performance will confirm the applicability of the corrected methods and therefore mental imagery on learning the task. This phase ends when mental imagery can be practiced effectively and the tasks practiced are of the same connectionist network and of similar difficulty level as those prospective tasks that may be experienced by the individual when discharged to a community setting, such as "taking a bath", "going to the toilet", "using the telephone" and "operating an automatic teller machine". For individuals who can independently maintain an upright position to enter TGT, the proposed duration of the programme is three weeks, with five one-hour sessions for five days per week.

After discharge to the community, mental imagery is effective in helping individuals to overcome the problems that may arise in new tasks that have not been practiced since sustaining damage to the brain. Individuals are encouraged to practice mental imagery before actual task performance. This facilitates the generalisation of the skills learnt in training institutions to the community setting. Guidance on the solutions to the problems and the effective use of mental imagery is given by rehabilitation personnel. The target is to help the individuals to establish mental imagery as a habit in their lives until they become more competent at meeting the ever-changing challenges of daily life.

\section{Conclusion}

Current clinical practice does not focus on the generalisation of skills trained to the needs of future community living. Individuals may face difficulties in performing those tasks which are not practiced in the hospital and rehabilitation settings. The current paper reviews the theory and a treatment programme for people with brain damage that promotes the generalisation of the skills relearnt. The Connectionist Model stipulates the shared structure of similar connected tasks. With the tasks under the same connectionist network, training on part of the tasks helps to reestablish the connection weights of the damaged units and the connections of those tasks, as well as promoting the relearning of the other tasks. Therefore, with the concept of task generalisation, training strategies should be geared towards the connectionist model, enhancing individual's ability to adapt to the needs of their environment in their future lives. This approach provides multidisciplinary involvement in treating people with brain damage with a theoretical base. Since the training programme allows generalisation of skills learnt to other tasks that are not trained during the rehabilitation phase, improved re-integration to the community is facilitated. This also helps to reduce the scope and time spent on strategies using specific task training under the current clinical practice. The positive results obtained in this study will provide the ground work for further development in task training to enable people with brain damage or other clinical conditions to relearn tasks.

\section{References}

1. Hoenig H, Horner RD, Duncan PW, Clipp E, Hamilton B (1999) New horizons in stroke rehabilitation research. J Rehabil Res Dev 36: 19-31.

2. Mauthe RW, Haaf DC, Hayn P, Krall JM (1996) Predicting discharge destination of stroke patients using a mathematical model based on six items from the Functional Independence Measure. Archives of Physical Medicine and Rehabilitation 77: 10-13.

3. Wentworth DA, Atkinson RP (1996) Implementation of an acute stroke program decreases hospitalization costs and length of stay. Stroke 27: 1040-1043.

4. Wright RE, Rao N, Smith RM, Harvey RF (1996) Risk factors for death and emergency transfer in acute and subacute inpatient rehabilitation. Arch Phys Med Rehabil 77: 1049-1055.

5. Grube MM, Dohle C, Djouchadar D, Rech P, Bienek K, et al. (2012) Evidence-based quality indicators for stroke rehabilitation. Stroke 43: 142-146.

6. Müller M, Strobl R, Grill E (2011) Goals of patients with rehabilitation needs in acute hospitals: goal achivement is an indicator for improved functioning. J Rehabil Med 43: 145-150.

7. Sabel BA (1999) Restoration of vision I: neurobiological mechanisms of restoration and plasticity after brain damage - a review. Restor Neurol Neurosci 15: 177-200.

8. Chan KC, Fu Q-L, Hui ES, So K-F, Wu EX (2008) Evaluation of the Retina and Optic Nerve in a Rat Model of Chronic Glaucoma using in vivo Manganese-enhanced Magnetic Resonance Imaging. NeuroImage 40: $1166-1174$

9. Gaffan D, Eacott MJ (1995) Visual learning for an auditory secondary reinforcer by macaques is intact after uncinate fascicle section: Indirect 
evidence for the involvement of the corpus striatum. European Journal of Neuroscience 9: 3144-3150.

10. Jones TA, Chu CJ, Grande LA, Gregory AD (1999) Motor skills training enhances lesion-induced structural plasticity in the motor cortex of adult rats. J Neurosci 19: 10153-10163.

11. Roiha K, Kirveskari E, Kaste M, Mustanoja S, Mäkelä JP, et al. (2011) Reorganization of the primary somatosensory cortex during stroke recovery. Clin Neurophysiol 122: 339-345.

12. Mahncke HW, Connor BB, Appelman J, Ahsanuddin OB, Hardy JL, et al. (2006) Memory enhancement in healthy older adults using a brain plasticity-based training program: A randomized, controlled study. Proceedings of the National Academy of Sciences 103: 12523-12528.

13. Jones TA, Hawrylak N, Klintsova AY, Greenough WT (1998) Brain damage, behaviour, rehabilitation, recovery, and brain plasticity. Mental Retardation and Developmental Disabilities Research Reviews 4: 231-237.

14. Rand D, Givon N, Weingarden H, Nota A, Zeilig G (2014) Eliciting Upper Extremity Purposeful Movements Using Video Games: A Comparison With Traditional Therapy for Stroke Rehabilitation. Neurorehabil Neural Repair.

15. Bussey TJ, Saksida LM (2002) The organization of visual object representations: a connectionist model of effects of lesions in perirhinal cortex. European Journal of Neuroscience 15: 355-364.

16. Atkins PWB, Murre JMJ (1998) Recovery of unrehearsed items in connectionist models. Connection Science 10: 99-119.

17. Plaut DC (1996) Relearning after damage in connectionist networks: toward a theory of rehabilitation. Brain Lang 52: 25-82.

18. Shallice $\mathrm{T}$ (2014) On compensatory strategies and computational models: The case of pure alexia. Cogn Neuropsychol 31: 529-543.

19. Takac M, Benuskova L, Knott A (2012) Mapping sensorimotor sequences to word sequences: a connectionist model of language acquisition and sentence generation. Cognition 125: 288-308.

20. Read SJ, Miller LC (2013) Connectionist models of social reasoning and social behaviour. Taylor and Francis Group, New York.

21. Cannon HM, Feinstein AH, Friesen DP (2010) Managing Complexity: Applying the Conscious-Competence Model to Experiential Learning. Developments in Business Simulation and Experiential Learning 37: 172-182.

22. Bloom BS, Madaus GF, Hastings JT, (1981) Evaluation to improve learning. McGraw-Hill, New York.

23. Langkvist M, Karlsson L, Loutfi A (2014) A review of unsupervised feature learning and deep learning for time-series modeling. Pattern Recognition Letters 42: 11-24.

24. Bechtel W, Abrahamsen A (1991) Connectionism and the Mind: An introduction to parallel processing in networks. Basil Blackwell, Cambridge, MA.

25. Bandura A (1986)Social foundations of thought and action: A social cognitive theory. Prentice-Hall, Englewood Cliffs, NJ.

26. McKeough A, Lupart JL, Marini A (2013) Teaching for transfer: Fostering generalisation in learning. Routledge, New York.

27. Liu KP, Chan CC, Lee TM, Hui-Chan CW (2004) Mental imagery for promoting relearning for people after stroke: a randomized controlled trial. Arch Phys Med Rehabil 85: 1403-1408.
28. Liu KP, Chan CC, Wong RS, Kwan IW, Yau CS, et al. (2009) A randomized controlled trial of mental imagery augment Generalisation of learning in acute poststroke patients. Stroke 40: 2222-2225.

29. Yamaguchi M, Logan GD (2014) Pushing typists back on the learning curve: revealing chunking in skilled typewriting. J Exp Psychol Hum Percept Perform 40: 592-612.

30. Liu KP, Chan CC, Lee TM, Hui-Chan CW (2004) Mental imagery for relearning of people after brain injury. Brain Inj 18: 1163-1172.

31. Byrnes JP (2013) The nature and development of decision-making: A self-regulation model. Psychology Press.

32. Liu KP, Chan CC2 (2014) Pilot randomized controlled trial of selfregulation in promoting function in acute poststroke patients. Arch Phys Med Rehabil 95: 1262-1267.

33. Liu KP, Chan CC, Lee TM, Li LS, Hui-Chan CW (2002) Self-regulatory learning and Generalisation for people with brain injury. Brain Inj 16: 817-824.

34. Bläsing B, Calvo-Merino B, Cross ES, Jola C, Honisch J, et al. (2012) Neurocognitive control in dance perception and performance. Acta Psychol (Amst) 139: 300-308.

35. Richards CA, Sanderson JA (1999) The role of imagination in facilitating deductive reasoning in 2-, 3- and 4-year-olds. Cognition 72: B1-9.

36. Hoyek N, Di Rienzo F, Collet C, Creveaux T, Guillot A (2014) Hand mental rotation is not systematically altered by actual body position: Laterality judgment versus sameâ $€$ "different comparison tasks. Attention, Perception, \& Psychophysics 76: 519-526.

37. Schieber MH (2014) Constraints and Flexibility in Cortical Control of the Hand, in The Human Hand as an Inspiration for Robot Hand Development, R Balasubramanian, VJ Santos, Editors. Springer International Publishing, Switzerland 3-21.

38. Debarnot U, Sperduti M2, Di Rienzo F3, Guillot A4 (2014) Experts bodies, experts minds: How physical and mental training shape the brain. Front Hum Neurosci 8: 280.

39. Saj A, Raz N, Levin N, Ben-Hur T, Arzy S (2014) Disturbed Mental Imagery of Affected Body-Parts in Patients with Hysterical Conversion Paraplegia Correlates with Pathological Limbic Activity. Brain Sciences 4: 396-404.

40. Parsons LM, Fox PT, Downs JH, Glass T, Hirsch TB, et al. (1995) Use of implicit motor imagery for visual shape discrimination as revealed by PET. Nature 375: 54-58.

41. Allali G, van der Meulen M, Beauchet $\mathrm{O}$, Rieger SW, Vuilleumier P, et al. (2013) The Neural Basis of Age-Related Changes in Motor Imagery of Gait: An fMRI Study. J Gerontol A Biol Sci Med Sci .

42. Kober SE, Wood G2 (2014) Changes in hemodynamic signals accompanying motor imagery and motor execution of swallowing: a near-infrared spectroscopy study. Neuroimage 93 Pt 1: 1-10.

43. Garrison KA, Winstein CJ, Aziz-Zadeh L (2010) The mirror neuron system: a neural substrate for methods in stroke rehabilitation. Neurorehabil Neural Repair 24: 404-412.

44. Guillot A, Di Rienzo F, MacIntyre T, Moran A, Collet C (2012) Imagining is not doing but involves specific motor commands: a review of experimental data related to motor inhibition. Frontiers in Human Neuroscience 6: 247 . 